\title{
De novo transcriptome characterization and gene expression profiling of the desiccation tolerant moss Bryum argenteum following rehydration
}

\author{
Bei Gao ${ }^{1,2}$, Daoyuan Zhang ${ }^{1 *}$, Xiaoshuang Li', Honglan Yang ${ }^{1}$, Yuanming Zhang ${ }^{1}$ and Andrew J. Wood ${ }^{3}$
}

\begin{abstract}
Background: The desiccation-tolerant moss Bryum argenteum is an important component of the Biological Soil Crusts (BSCs) found in the Gurbantunggut desert. Desiccation tolerance is defined as the ability to revive from the air dried state. To elucidate the molecular mechanisms related to desiccation tolerance, we employed RNA-Seq and digital gene expression (DGE) technologies to study the genome-wide expression profiles of the dehydration and rehydration processes in this important desert plant.
\end{abstract}

Results: We applied a two-step approach to investigate the gene expression profile upon rehydration in the moss Bryum argenteum using Illumina HiSeq2000 sequencing platform. First, a total of 57,247 transcript assembly contigs (TACs) were obtained from 54.79 million reads by de novo assembly, with an average length of 863 bp and N50 of 1,372 bp. Among the reconstructed TACs, 36,916 (64.5 \%) revealed similarity with existing protein sequences in the public databases. 23,509 and 21,607 TACs were assigned GO and KEGG annotation information, respectively. Second, samples were taken from 3 hydration stages: desiccated (Dry), rehydrated $2 \mathrm{~h}$ (R2) and rehydrated $24 \mathrm{~h}$ (R24), and DEG libraries were constructed for Differentially Expressed Genes (DEGs) discovery. 4,081 and 6,709 DEGs were identified in R2 and R24, compared with Dry, respectively. Compared to the desiccated sample, up-regulated genes after two hours of hydration are primarily related to stress responses. GO function enrichment network, EKGG metabolic pathway and MapMan analysis supports the idea of the rapid recovery of photosynthesis after $24 \mathrm{~h}$ of rehydration. We identified 770 transcription factors (TFs) which were classified into 50 TF families. 142 TF transcripts were up-regulated upon rehydration including 23 members of the ERF family.

Conclusions: In this study, we constructed a pioneering, high-quality reference transcriptome in B. argenteum and generated three DGE libraries to elucidate the changes of gene expression upon rehydration. Expression profiles consistent with the rapid recovery of photosynthesis (at R2) and the re-establishment of a positive carbon balance following rehydration (at R24) were observed. Our study will extend our knowledge of bryophyte transcriptomes and provide further insight into the molecular mechanisms related to rehydration and desiccation-tolerance.

Keywords: Transcriptome, Gene expression, Desiccation, Bryum, Physcomitrella, Biological soil crust

\section{Background}

Desiccation tolerance (DT) is characteristic of many organisms $[1,2]$. DT is a common feature of the land plant life cycle and is often restricted to reproductive structures such as seeds and spores [3, 4]. DT of vegetative tissues such as leaves is rare in angiosperms [3] and common in mosses

\footnotetext{
* Correspondence: zhangdy@ms.xjb.ac.cn

${ }^{1}$ Key Laboratory of Biogeography and Bioresource in Arid Land, Xinjiang Institute of Ecology and Geography, Chinese Academy of Sciences, Urumqi 830011, China

Full list of author information is available at the end of the article
}

[5]. In many arid and semiarid ecosystems, vegetative cover is sparse. The open spaces in such areas are covered by desiccation-tolerant mosses, cyanobacteria, fungi, green algae and lichens, which are the main components of biological soil crusts (BSCs) [6, 7]. In arid regions, BSCs play a number of important ecological roles which include reducing soil erosion, improving water infiltration and contributing to carbon assimilation and nitrogen fixation [6, 7]. The Gurbantunggut desert (Xinjiang, China) is one of the major arid regions of central Asia and is the largest fixed and semi-fixed cold desert in China. BSCs of 
the Gurbantunggut desert are dominated by two moss species: Syntrichia caninervis and Bryum argenteum [7]. BSCs of the Tengger desert (found in northwest China) are dominated by $B$. argenteum $[8,9]$. In the desert environment, moss crusts are frequently subjected to cycles of dehydration, desiccation and rehydration [10]. Organisms found in BSCs have developed a suite of adaptive mechanisms that permit the avoidance of water loss and/or the survival of complete dehydration (i.e. desiccation) [10, 11].

More than 200 moss species have been experimentally verified to be desiccation-tolerant and Tortula ruralis (= Syntrichia ruralis) is the model species for understanding the molecular aspects of vegetative desiccation-tolerance in mosses $[1,2,4]$. B. argenteum, like S. caninervis and $T$. ruralis, is classified as the most desiccation-tolerant moss species, able to survive equilibration with extremely dry air (i.e. $0-30 \% \mathrm{RH}$ ) which could tolerant to $30 \% \mathrm{RH}$ or below [5]. A common feature of desiccation-tolerant mosses is the rapid recovery of photosynthesis and the rapid reestablishment of a positive carbon balance following rehydration [12]. Previous study suggested that on rehydration of the dry bryophytes, both the photosynthetic electron flow and the PSII activity recovered quickly in B. argenteum [13]. Similar to other desiccation-tolerant mosses, recent studies suggested that the thylakoid protein complexes of $B$. argenteum degrade during dehydration and then reassemble following rehydration, and synthesis of proteins, at least those involved in thylakoid protein complexes, was required for the full recovery of photosynthesis after rehydration [11].

In this study, we employed next generation Illumina sequencing technology to investigate the $B$. argenteum transcriptome and conduct comparisons of the digital gene expression profiles. This study was performed based on a two-step approach: (a) Generating and annotating the integrated transcriptome of $B$. argenteum, and (b) Constructing digital gene expression (DGE) libraries to identify differentially expressed genes (DEGs) between the desiccated (Dry) and subsequently rehydrated (R2 and R24) gametophytes. To our knowledge this is the first description of the $B$. argenteum transcriptome and one of the few annotated bryophyte transcriptomes. Our study extends our knowledge of bryophyte transcriptomes and provides further insight into the molecular mechanisms related to rehydration and desiccation-tolerance.

\section{Results and discussion}

\section{Transcriptome sequencing and de novo assembly}

In the absence of a reference genome for Bryum argenteum, we assembled a de novo reference transcriptome for reads mapping and gene expression profiling. To obtain a comprehensive transcriptome in $B$. argenteum and an overview of its gene expression profiles at various dehydrated/rehydrated stages, an equal mixture of total RNAs isolated from various dehydration and rehydration time points were used to construct the sequencing library. Gametophores were harvested from three different time points: desiccated (Dry), rehydrated for $2 \mathrm{~h}$ (R2) and rehydrated for $24 \mathrm{~h}$ (R24). Using the Illumina NGS platform HiSeq2000, approximately 62.11 million raw sequencing reads were obtained, and 54.79 million paired 90 bp clean reads were obtained after adapter and low-quality reads trimming.

With the Trinity de novo assembler 106,066 contigs were produced, including different isoforms per contig. To obtain all non-redundant consensus transcript sequences, these initially assembled contigs were clustered using the TGICL to generate the final transcript assembly contigs (TACs hereafter). The statistical characteristics of these contigs and final TACs were shown in Table 1. All reconstructed TACs longer than or equal to $200 \mathrm{bp}$ were retained for further analysis. A total of 57,247 TACs were represented by 14,108 distinct clusters and 43,139 singletons. The longest TAC has a length

Table 1 Summary of the B. argenteum transcriptome assembly and annotation

\begin{tabular}{|c|c|c|}
\hline \multirow[t]{6}{*}{ Reads } & Total Raw Reads & $62,112,418$ \\
\hline & Total Clean Reads & $54,787,000$ \\
\hline & Clean Nucleotides (nt) & $4,930,830,000$ \\
\hline & Reads length (nt) & $90 \times 2$ \\
\hline & Q20 percentage & $98.67 \%$ \\
\hline & GC percentage & $52.05 \%$ \\
\hline \multirow[t]{4}{*}{ Contigs } & Total Number & 106,066 \\
\hline & Total Length (nt) & $43,542,237$ \\
\hline & Mean Length (nt) & 411 \\
\hline & N50 (nt) & 908 \\
\hline \multirow[t]{10}{*}{ Unigenes } & Total Number & 57,247 \\
\hline & Distinct Clusters & 14,108 \\
\hline & Distinct Singletons & 43,139 \\
\hline & Total Length (nt) & $49,383,291$ \\
\hline & Mean Length (nt) & 863 \\
\hline & N50 (nt) & 1,372 \\
\hline & L50 number & 11,094 \\
\hline & Min Unigene Length (nt) & 200 \\
\hline & Max Unigene Length (nt) & 14,247 \\
\hline & Average Depth $(\times)$ & 85.38 \\
\hline \multirow[t]{6}{*}{ Annotation } & NCBI-nr & $35,813(62.6 \%)$ \\
\hline & Swiss-Prot & 20,864 (39.9\%) \\
\hline & KEGG & 21,607 (37.7 \%) \\
\hline & COG & 16,715 (29.2 \%) \\
\hline & $\mathrm{GO}$ & 23,509 (41.1\%) \\
\hline & All & $36,446(63.7 \%)$ \\
\hline
\end{tabular}


of 14,247 bp. The final retained TAC set has an average length of $863 \mathrm{bp}$ and N50 of 1,372 bp. The abundance of TACs were evaluated relative to length (Fig. 1a) and sequencing depth (Fig. 1b), respectively. An average sequencing depth of $85 \times$ for the final transcriptome assembly was achieved. We then used the $P$. patens reference genome $[14,15]$ and the ortholog hit ratio method [16] to evaluate the $B$. argenteum TAC integrity, the result indicated that more than $95 \%$ of the putative $P$. patens orthologs captured within the $B$. argenteum transcriptome covered at least $90 \%$ of the predicted ORF (Additional file 1: Figure S1). The 357 Arabidopsis thaliana single copy genes conserved across all eukaryotes (UCOS, see Methods) was used to further assess the completeness of transcriptome. As a result, the $B$. argenteum transcriptome assembly contained 352 putative homologs of the 357 Arabidopsis thaliana UCOS proteins. And similar results were also obtained for the newly sequenced Ceratodon purpureus transcriptome [17]. This suggested that we have assembled and captured an intact transcriptome with respect to transcript integrity and trancriptome completeness. Based upon these metrics, this $B$. argenteum transcriptome is of higher quality than the $S$. caninervis transcriptome reported previously by our group [18].

\section{Functional annotation of $B$. argenteum transcriptome}

To identify the putative functions of TACs related to the rehydration process, a sequence similarity search was carried out against protein sequences available at NCBInr, Swiss-Prot, KEGG and COG databases using BLASTX algorithm with an E-value threshold of 1e-5 (Additional file 2). Among the 57,247 reconstructed TACs, similarity with known proteins was detected for 36,446 TACs which represent $64 \%$ of the de novo reference transcriptome assembly (Table 1 and Additional file 1: Figure S2). Among the TACs that returned a positive BLASTX hit within the NCBI-nr database, more than $70 \%$ of the best aligned sequences were shown to be derived from the model moss P. patens (Additional file 1: Figure S3). Similar results have been depicted for the desert moss S. caninervis [18]. Many TACs could not be matched to a protein sequence within the databases and therefore lack a functional description. Lack of sequence identity with known proteins is partially explained by the limited genomic data available for mosses. To date, the P. patens genome is the only moss reference genome [14]. Unmatched sequences could be orphan genes, non-coding RNA or sequences from UTR regions, and we cannot exclude the possibility of partially or misassembled transcripts.

For further descriptive analysis, we divided our transcriptome TAC set into two subsets: TACs with detected protein homology ('with BLAST') and TACs without protein homology ('without BLAST'). The length of longest open reading frames (ORF) and the protein coding potential score of all TACs were determined by CPAT software [19]. Boxplot of the TACs and ORF length of the two sets is depicted in Fig. 2a. The 'with BLAST' group (average length $=1,124 \mathrm{bp}$ ) had larger TAC sizes than the 'without BLAST' (average length $=405 \mathrm{bp}$ ), and same to the ORF sizes (mean length of 'with BLAST' and 'without BLAST' groups were $730 \mathrm{bp}$ and $209 \mathrm{bp}$, respectively). Furthermore, higher protein coding potential score, higher GC content and higher expression levels were all encountered for the 'with BLAST' group (Fig. 2b-d). Results similar to those obtained for the 'with BLAST' have been reported for the wasp, which is also a species without a reference genome [20]. The characteristics of TACs lacking homology - shorter ORFs, lower protein coding potential, lower GC content and lower expression values - may suggest that a large proportion of novel TACs correspond to non-protein coding
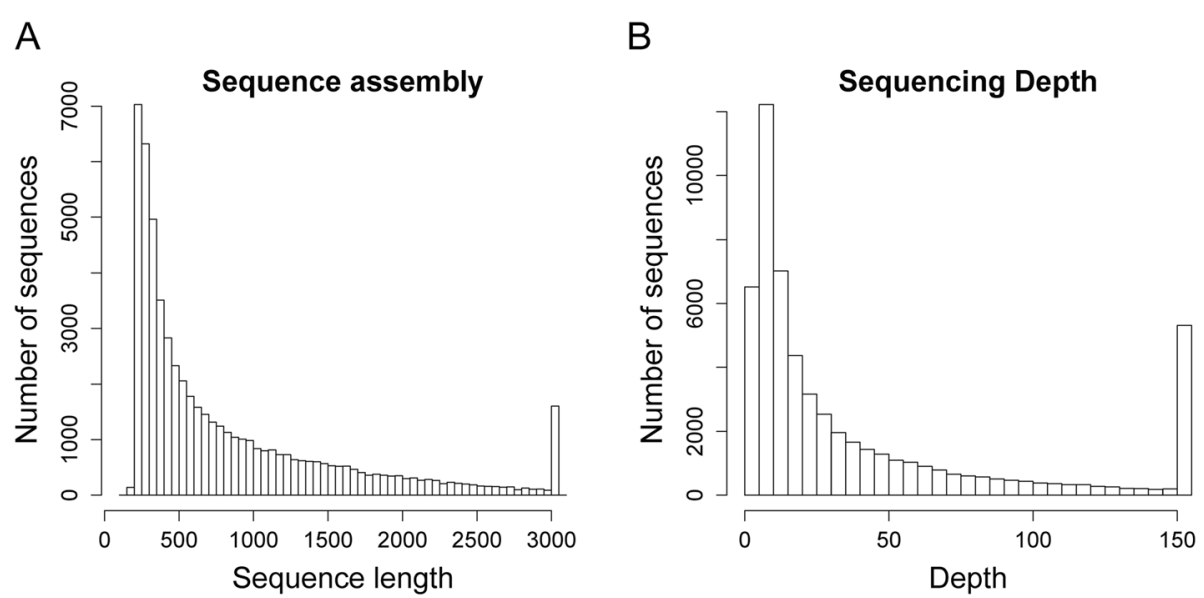

Fig. 1 Overview of the B. agenteum transcriptome sequencing and assembly. Histogram of the length (a) and sequencing depth (b) of transcript assembled contigs 
A

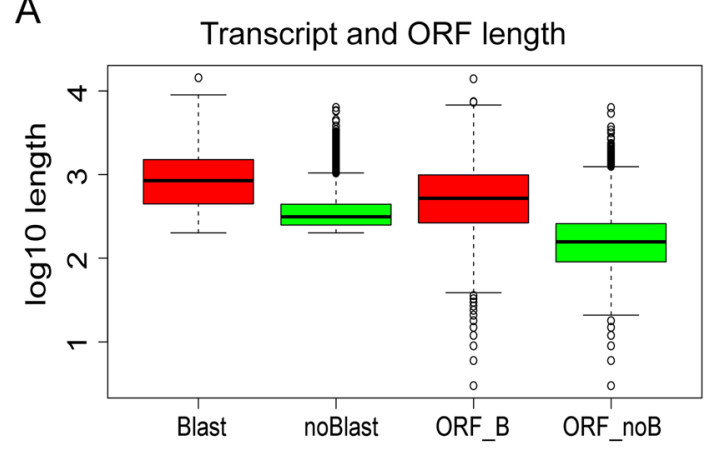

B

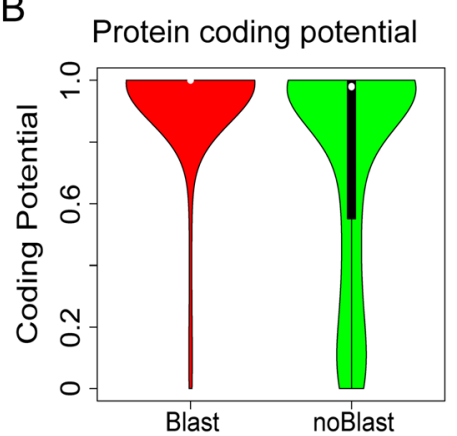

C

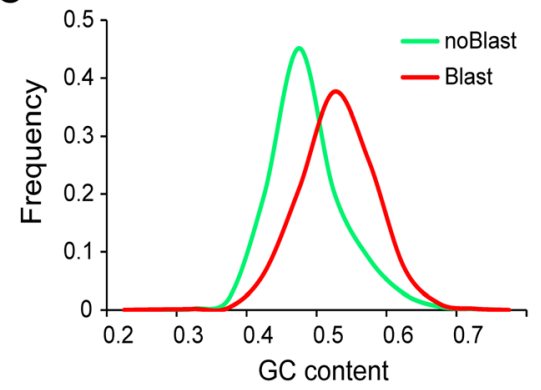

D

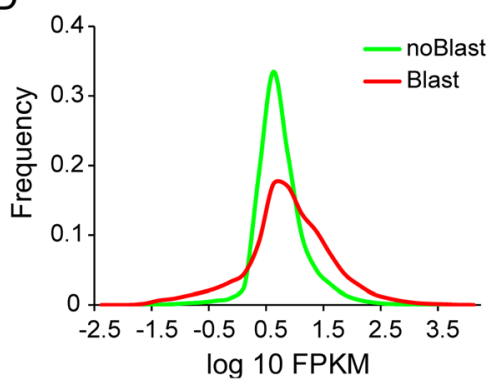

Fig. 2 Sequence and expression characterization of the transcripts with and without detected homologs. (a) Overall length and ORF length, (b) Protein coding potential determined by CPAT, (c) distribution of GC content, (d) Overall normalized expression level

transcripts [21]. But low sequence conservation across species, lacking of genomic information for desert mosses and the typical tissue specificity [21] of the non-coding RNAs may also explain the novelty of these genes and prevent their annotation.

To understand the putative functional categories of the annotated TACs, gene ontology (GO) was employed to classify the annotated transcripts. Of the 36,466 TACs with significant similarity to NCBI-nr proteins, 23,509 (64\%) were assigned to $\mathrm{GO}$ terms (Fig. 3) and the widely used gene Clusters of Orthologous Groups (COG) functional classification result was illustrated in Additional file 1: Table S1. Previous GO annotation in the mosses $S$. caninervis and $P$. patens assigned a functional annotation of $48 \%$ [18] and $58 \%$ [15], respectively. GO comparison between $S$. caninervis, $P$. patens and $B$. argenteum demonstrated similar sequence enrichment across all three GO categories and is consistent with previous results [18]. For all three moss species transcripts were enriched within the Cellular Component category ("cell", "cell part" and "organelle"), the Molecular Function category ("binding" and "catalytic") and the Biological Processes category ("cellular process" and "metabolic processes"). GO functional categories such as "symplast", "biological regulation", "cellular component organization", "cellular processes", "developmental processes", "multicellular organismal process" and "response to stimulus" are significantly more represented in B. argenteum as compared to the other two mosses.

Kyoto Encyclopaedia of Genes and Genomes (KEGG) provides a basic platform for the systematic analysis of gene function in terms of the metabolic networks of gene products [22]. For further understanding of the biological functions and interactions of genes, pathwaybased analysis was conducted based on the KEGG Pathway database. TACs annotated by KEGG orthology proteins were mapped to the metabolic pathways. A total of 21,607 TACs were mapped to 127 reference canonical pathways. The annotations and classifications for these TACs provided a resource for investigating specific pathways in B. argenteum, such as photosynthetic carbon reduction and cell wall biosynthesis.

Identifying conserved domains present within a deduced polypeptide can provide insight into the function, regulation and/or localization of the predicted protein. The deduced polypeptide sequences from the B. argenteum transcriptome were queried for the presence of protein motifs using the Pfam database [23]. 23,654 deduced polypeptide sequences were assigned Pfam domain/ family information and categorized into 4,091 Pfam families (Additional file 3). Among the sequences with a detectable Pfam domain, 22,600 (95.5\%) sequences had homology sequences ('with BLAST') in public protein 


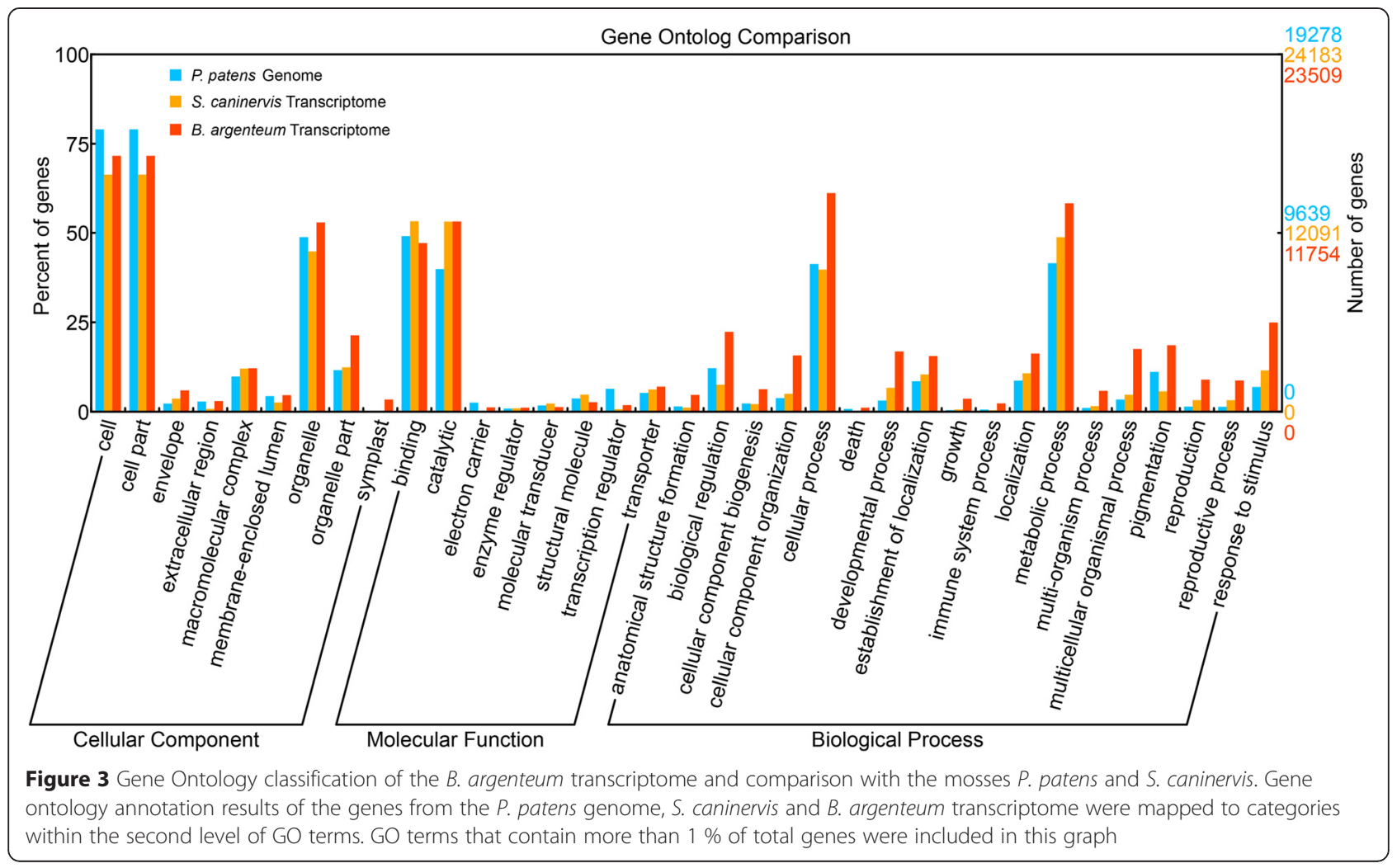

databases, and 1,054 (4.5\%) sequences had no BLASTX hits ('without BLAST') but protein domains could be detected using HMMER. Then Pfam domains/families were ranked according to the frequency of occurrence of $B$. argenteum TACs. And among the identified protein domains/families, 'Pkinase' and its subclass 'Pkinase-Tyr', 'WD40', 'RRM_1' and 'ABC-tran' were the most abundant ones (Additional file 3), similar results was also reported for the $S$. caninervis transcriptome [18].

Transcription factors (TFs) play key roles in plant development and stress response by temporarily and spatially regulating the transcription of their target genes. In this study, we conducted a comprehensive annotation and classification of the TFs in B. argenteum. A total of 770 TACs were identified and classified into 50 TF families (Additional file 4) according to the family assignment rules illustrated in PlantTFDB v3.0 [24]. ERF and bHLH TF families revealed to be the two largest TF families, consisting of 91 and 63 gene family members, respectively.

\section{Expression analysis and identification of differentially expressed genes}

To investigate the digital gene expression profiles of the desiccated and rehydrated gametophores, three digital gene expression (DGE) libraries (Dry, R2 and R24, see Methods) were constructed and sequenced using Illumina deep sequencing technology. More than 3.4 million clean reads were obtained from each library and then aligned to the reference transcriptome separately. Approximately, $58 \%$ (Dry), $55 \%$ (R2) and $50 \%$ (R24) reads mapped uniquely to the reference transcriptome assembly, and 15-19\% of reads were filtered as multiplealigned and ignored in subsequent analyses (Table 2). The three DGE libraries shared a total of 28,569 TACs, accounting for $50 \%$ of the reference transcriptome assembly, and 1,433 (Dry), 3140 (R2) and 2,872 (R24) TACs were putatively uniquely expressed. A comparison between Dry and the rehydrated DGE libraries (R2 and R24) indicated that $96 \%$ of genes detected in the Dry library were also detected in either one or both of the rehydrated libraries (Fig. 4a). The number of reads aligned to a TAC ranged from 1 to 22,846, with a mean of 62.2 for Dry, 46.5 for R2 and 42.9 for R24. Violin plots for the number of reads aligned to a TAC are depicted in Fig. 4b. Based on the number of uniquely aligned reads, each TAC was assigned with a RPKM value [25], which could normalize for the total reads obtained in each individual library (Additional file 5). Based on this analysis, the gene expression levels in desiccated and rehydrated libraries were classified into five categories (rare, low, moderate, high and extremely high) (Fig. 4c). The largest portion of TACs in Dry exhibited rare expression (RPKM $<3$ ). However, the low expressed TACs (RPKM > 3-10) occupied the biggest proportion in the rehydrated libraries. Only a small fraction 
Table 2 Summary for DGE sequencing datasets

\begin{tabular}{llll}
\hline Summary & Dry & R2 & R24 \\
\hline Total clean reads & $3,666,367$ & $3,488,408$ & $3,488,106$ \\
Aligned reads (N) & $2,824,311$ & $2,453,703$ & $2,278,641$ \\
Aligned reads (\%) & $77.03 \%$ & $70.34 \%$ & $65.33 \%$ \\
Perfect aligned reads (N) & $2,500,618$ & $2,150,062$ & $2,000,264$ \\
Perfect aligned reads (\%) & $68.20 \%$ & $61.63 \%$ & $57.35 \%$ \\
Uniquely aligned reads (N) & $2,125,713$ & $1,908,260$ & $1,756,516$ \\
Uniquely aligned reads (\%) & $57.98 \%$ & $54.70 \%$ & $50.36 \%$ \\
Unigenes with uniquely aligned reads (N) & 34,161 & 41,023 & 40,902 \\
Unigenes with uniquely aligned reads (\%) & $59.67 \%$ & $71.66 \%$ & $71.45 \%$ \\
\hline
\end{tabular}

(3.7 \% to $3.9 \%$ ) of TACs was expressed at extremely high levels $(\mathrm{RPKM}>100)$. The average RPKM values of transcripts for Dry, R2 and R24 were 27.3, 24.1 and 23.8, respectively. Furthermore, functional classification of GO terms of the TACs detected from Dry, R2 and R24 were shown in Additional file 1: Figure S4.
Differentially expressed genes (DEGs) were determined by applying the screening thresholds of 2-fold changes and FDR $\leq 0.001$ [26]. Based on this analysis, the number of up-regulated DEGs were 3,255, 5,105 and 2,644, and down-regulated DEGs were 826, 1,604 and 1,850 in 'Dry v R2', 'Dry v R24' and 'R2 v R24', respectively (Fig. 4d-f). The
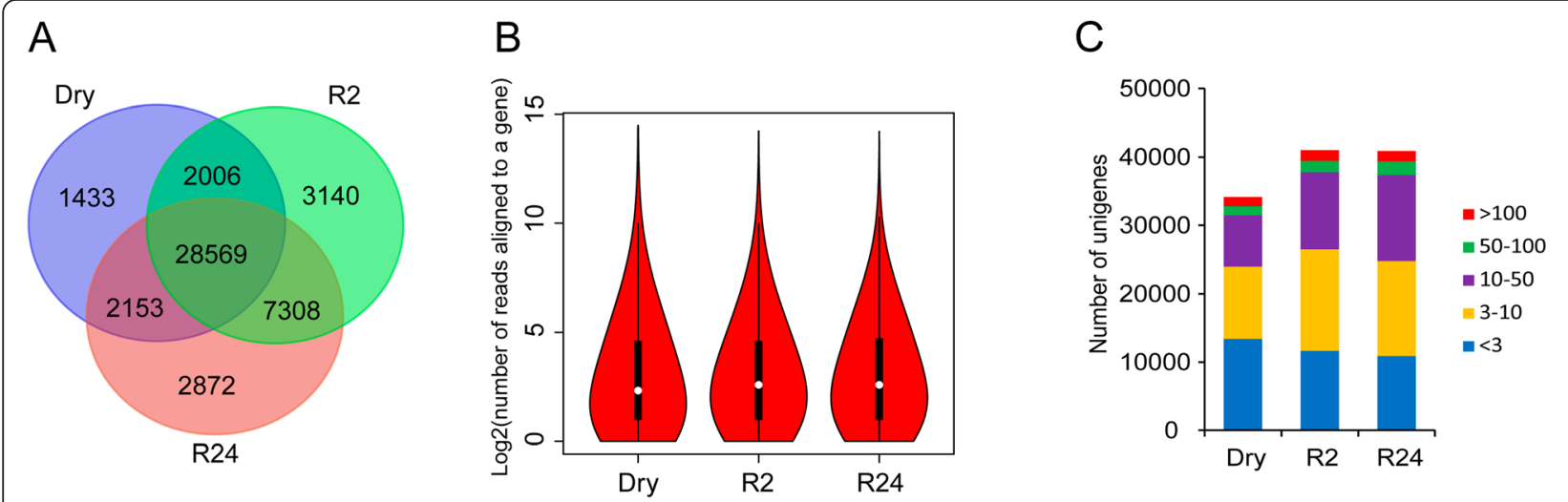

D

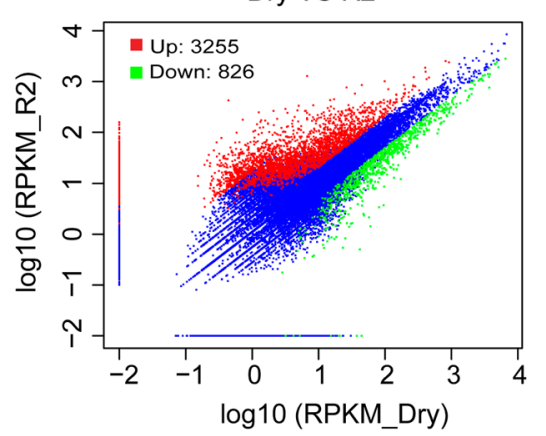

$\mathrm{E}$

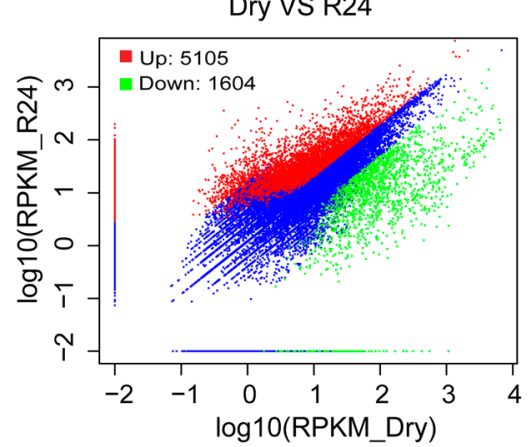

$\mathrm{F}$

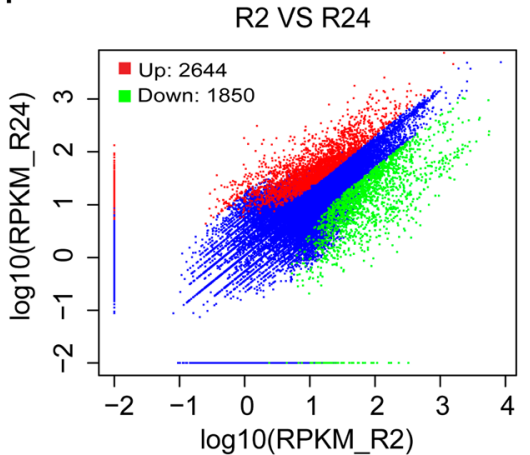

Fig. 4 Summary of DGE-seq mapping data and comparison of expressed transcripts between dehydrated and rehydrated samples. a Numbers of shared and unique TACs among dehydrated and rehydrated gametophores. $\mathbf{b}$ Violin plot for the number of reads uniquely mapped to a TAC. c Number of transcripts with different expression levels in dehydrated and rehydrated gametophores as measured by DGE sequencing. d-f The scatter plot comparing the gene expression levels pairwise among the three libraries (between Dry and R2, between Dry and R24, as well as between R2 and R24, respectively). The number of DEGs were also present in the figure 
ranking of up-regulated DEGs (from lowest to highest) is 'R2 v R24', 'Dry v R2' and 'Dry v R24' while the ranking of down-regulated DEGs is 'Dry v R2', 'Dry v R24' and 'R2 v R24'. Interestingly, 'Dry v R24' had the most up-regulated DEGs while 'R2 v R24' had the most down-regulated DEGs. These results suggest that the rehydration process is associated with the accumulation of novel transcripts, and that some transcripts are more abundant immediately after rehydration (i.e. R2) and are associated with an 'early response" to rehydration. All DEGs which exceed the threshold are listed in the Additional file 5.

Since TFs play crucial roles in the regulation of gene expression and plant stress response, we performed comprehensive analysis of the differentially expressed TFs. All annotated and differentially expressed B. argenteum TFs were listed in Additional file 4. The most upregulated TF after $2 \mathrm{~h}$ of rehydration was a MYB_related transcription factor (unigene20865_TBA), which contains a specific Myb_dna_bind domain (PF00249). The most up-regulated TF after $24 \mathrm{~h}$ of rehydration was a Ninlike transcription factor (unigene20858_TBA), which is recognized as a central role in nitrate signalling in $A$. thaliana [27]. Based upon TF family classification and DEG screening, the ERF is the most abundant TF in family in S. caninervis [18], P. patens $[15,24]$ and B. argenteum. Among all the characterized TF families, the $B$. argenteum ERF family contained the largest number of DEGs. 17 ERFs were up-regulated $2 \mathrm{~h}$ after rehydration and $23 \mathrm{ERFs}$ were up-regulated $24 \mathrm{~h}$ after rehydration. Among the 10 most up-regulated TFs, there are 4 ERFs and 3 GRAS transcription factors.

Studies have demonstrated that the AP2/ERF proteins have important functions in the transcriptional regulation of a variety of biological processes related to growth and development, as well as various responses to environmental stimuli [28]. The GRAS TF family was found throughout the plant kingdom, and these proteins have diverse roles in plant development, such as root and shoot development, gibberellic acid (GA) signalling and phytochrome A signal transduction [29]. The B. argenteum unigene9310_TBA is recognized as a DELLA protein, a subclass of the GRAS family, which may play important roles in gibberellic acid (GA) and light signalling [30], and was highly up-regulated $24 \mathrm{~h}$ after rehydration ('R24').

T. ruralis is a model moss for studying stress-responsive genes related to desiccation tolerance and rehydration, and a number of cDNAs/ESTs/transcripts have been isolated and characterized. We performed a BLASTX search against the $T$. ruralis protein sequences [31,32], obtained from NCBI protein database, using our $B$. argenteum TACs. A total of $236 \mathrm{~B}$. argenteum TACs were identified homologous (E-value $\leq 1 \mathrm{e}-5$ and identity $\geq 50 \%$ ) with the previously characterized $T$. ruralis transcripts, including
$\operatorname{Tr} 155$ [33] and $\operatorname{Tr} 288$ [34], Elipa and Elipb [35], ALDH7B6 [36], ALDH21A1 [37] and $\operatorname{TrDr} 3$ [38]. For instance, a $\operatorname{Tr} 155$ homolog detected in $B$. argenteum (unigene32501_TBA) was up-regulated upon rehydration, while the TrDr3 homologs (CL2407_TBA) were downregulated upon rehydration. A full list of the T. ruralis homologs detected in $B$. argenteum and their RPKM values were listed in Additional file 6.

\section{GO network analysis of DEGs upon rehydration}

To better understand the molecular mechanisms of rehydration $[1,4,5,31,32,35,36]$ and elucidate the $B$. argenteum rehydration process at the level of transcriptomics, DEG comparisons between the desiccated and rehydrated samples were performed. GO functional enrichment analysis for all DEGs from R2 ('R2 v Dry') and R24 ('R24 v Dry') were analyzed using BiNGO, a Cytoscape plugin assessing overrepresentation of ontologies in biological networks [39], using the list of all TACs from the reference transcriptome as background.

In order to clearly depict the GO enrichment network, we selected the collapsed GO-slim plant terms as namespace [40]. The collapsed GO enrichment network of the up-regulated TACs in R2 and R24 is shown in Fig. 5 and Additional file 1: Table S2, and the full GO term enrichment statistical results are shown in Additional file 7. For up-regulated TACs in R2, most of the GO terms were related to stress response, such as "response to stress", "response to abiotic stimulus" and "response to endogenous stimulus", which were also over-represented (corrected P-value $\leq$ 0.05) in R24 (Fig. 5). The GO terms "vacuole" and "plasma membrane" were significantly enriched in R2 (P-values of 7.56e-5 and 3.91e-3, respectively). In contrast to R2, the most over-represented upregulated R24 GO categories were "plastid", "thylakoid" and "photosynthesis" (P-values were 0, 3.24e-76 and $4.45 \mathrm{e}-60$, respectively). These results are consistent with the idea that alterations to membrane function and integrity is a short-term response (impacted by the in-rush of water) and that the recovery of photosynthesis is a long-term response. In both GO enrichment networks (i.e. R2 and R24), 'ribosome', 'translation' "cell wall", "cytosol" and "nucleolus" are over-representative GO terms. These results support the idea that translation plays a central role in desiccation tolerance $[41,42]$.

Following rehydration, the number of up-regulated DEGs was much larger than the number of downregulated DEGs, indicating the rehydration process was associated with accumulation of transcript rather than the non-accumulation of transcript (Fig. 4d-f). Due to the small number of down-regulated DEGs, we applied the full GO terms within the BiNGO namespace to perform GO functional enrichment analysis (Additional file 7). From the enrichment analysis, we identified a smaller 


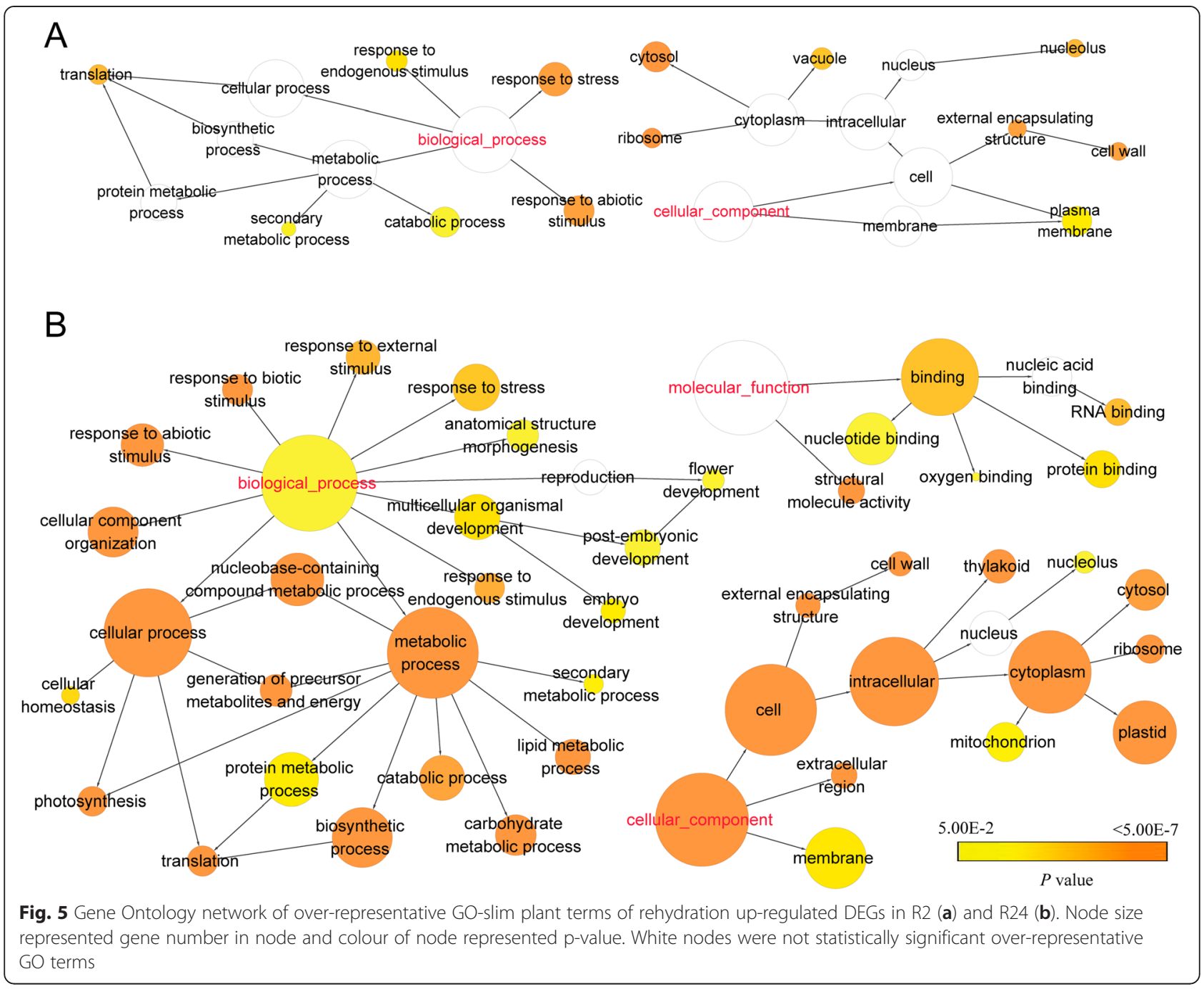

number of biologically informative $\mathrm{GO}$ terms as compared to the up-regulated DEGs. For down-regulated DEGs in R2, the GO terms of "transmembrane transport", "water transmembrane transporter activity", and "water channel activity" revealed to be the most significantly over-representative terms (P-values were 3.58E-04, 4.86E04 and 4.86E-04, respectively). Transcripts related to water transmembrane transport were less abundant upon rehydration. Concomitantly, transcripts related to 'response to water deprivation' and 'response to desiccation' were significantly down regulated $24 \mathrm{~h}$ after rehydration.

\section{Photosynthesis was significantly up-regulated upon full rehydration}

To identify the metabolic pathways that were significantly altered by rehydration, we used both KEGG pathway network and MapMan to uncover the altered metabolic pathways. Significantly altered KEGG pathways were identified using a P-value based on hypergeometric distribution. As a result, 15 and 23 metabolic pathways were identified to be significantly altered after $2 \mathrm{~h}$ and $24 \mathrm{~h}$ of rehydration, respectively (Fig. 6). KEGG pathway network analysis showed that "protein processing in ER", "ribosome", "proteasome" and "TCA cycle" were DEG-enriched in $B$. argenteum $2 \mathrm{~h}$ after of rehydration. Interestingly, the TCA cycle was only found to be enhanced within $2 \mathrm{~h}$ upon rehydration, suggesting the necessity of mitochondrial function in plant resuscitation.

The metabolic pathways "photosynthesis", "photosynthesis - antenna proteins" and "ribosome" were DEGenriched $24 \mathrm{~h}$ after rehydration. DEGs anchored on the pathways of photosynthesis, antenna proteins and ribosome are depicted in Additional file 1: Figure S5 - S7. All identified differentially expressed antenna proteins (Light Harvesting Complex, LHCs), including Lhca1, Lhca2, Lhca3 and Lhca5 in photosystem I and Lhcb1 - 6 in photosystem II were induced after $24 \mathrm{~h}$ of rehydration (Additional file 1: Figure S6). The majority of DEGs participating in photosynthesis and ribosome/translation (Additional file 1: Figure S7) were up-regulated TACs, 

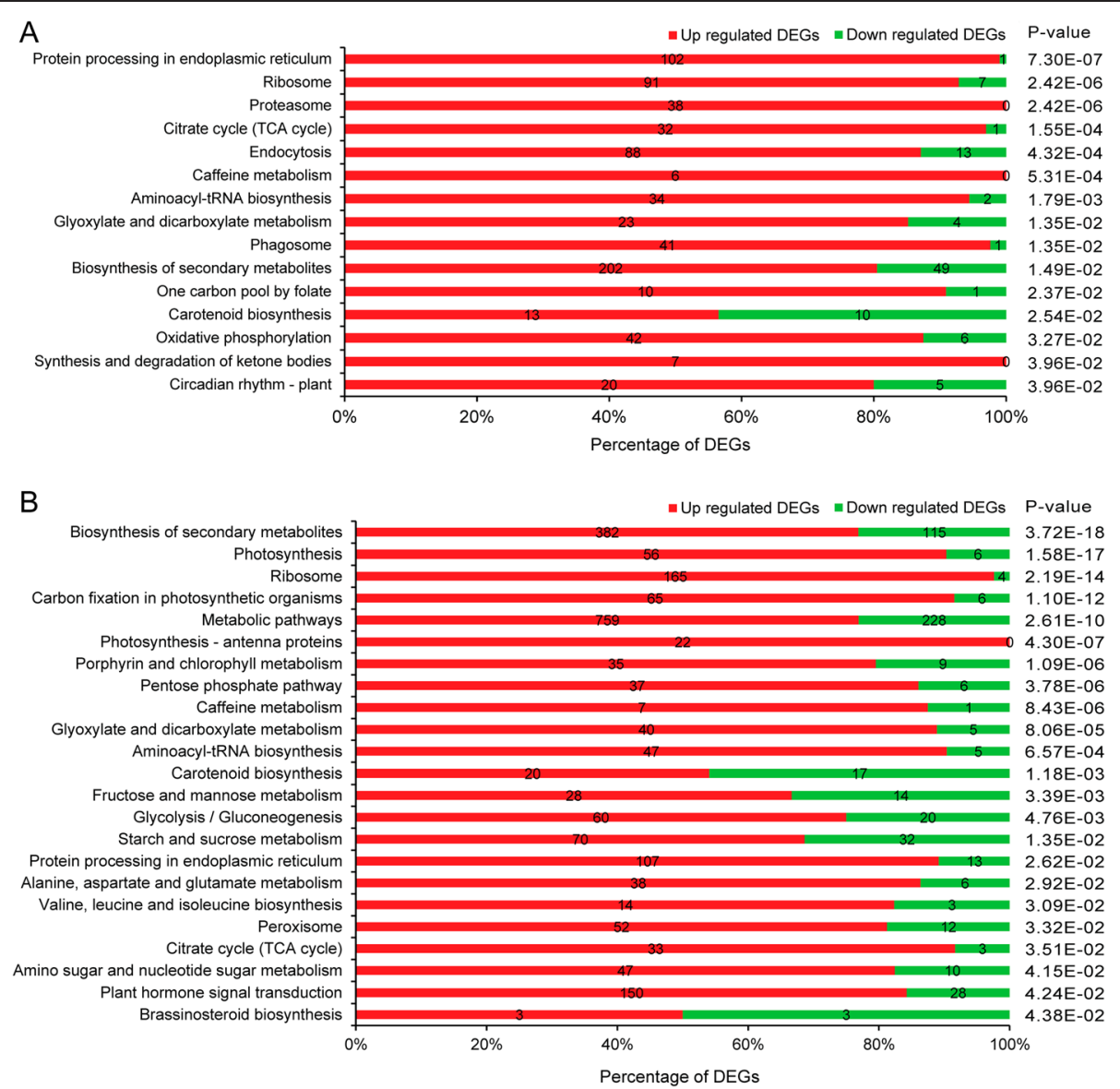

Fig. 6 Specific significantly enriched KEGG pathways for DEGs from R2 (a) and R24 (b). Number of up- and down-regulated DEGs was shown on the bar plot. The p-values shown for each pathway were FDR corrected

which is consistent with the GO enrichment network. So we could conclude the importance of translation from both GO and KEGG network enrichment analysis. Furthermore, we used MapMan to illustrate the altered expression of TACs for a number of metabolic pathways. In a MapMan comparison between R24 and Dry, TACs participating in "light reactions", "photorespiration" were up-regulated following $24 \mathrm{~h}$ of rehydration (Fig. 7). MapMan comparisons between Dry and R2, R2 and R24 were depicted in Additional file 1: Figure S8 and S9.

We were able to identify and quantify the expression of a number of $B$. argenteum transcripts related to photosynthesis. PsbS (unigene32903_TBA), Lhcb3 (unigene 18463_TBA, unigene18462_TBA, unigene11855_TBA), Lhc5 (CL4560 _TBA, unigene11018_TBA, unigene11017_ TBA) and Lhcb6 (unigene29917_TBA) were up-regulated $24 \mathrm{~h}$ after rehydration. Lhc proteins are fundamental to light-harvesting and PsbS (a Lhc-like protein) is thought to play a key role in NPQ [43]. Lhca4 (unigene40215 TBA) was identified in $B$. argenteum but was not differentially expressed. In P. patens, Lhc4 has not been identified and Lhc5 does not accumulate under standard growth conditions [44]. The presence of the Lhcb3 and Lhcb6 protein families are limited to land plants and are postulated to play a role in the adaptation to aerial environments [45]. Previous research in T. ruralis has demonstrated that the Elip transcripts accumulate in response to desiccation, salinity and high light, and are down-regulated upon rehydration [35]. Elips are postulated to scavenge free $\mathrm{Chl} a$ and protect PSII from photo-oxidative damage. The gene expression profile for $B$. argenteum Elips is depicted in Additional file 1: Figure S10. Consistent with T. ruralis, Elip genes are associated with desiccated tissue and are down-regulated $24 \mathrm{~h}$ after rehydration.

\section{Quantitative real-time PCR validation}

Quantitative RT-PCR (qPCR) analysis was performed to test the reliability of the data generated using the next generation sequencing platform. We selected 12 representative TACs based upon their hypothesized role(s) in desiccation tolerance. We validated 5 transcription factors (i.e. ERF, NAC, GRAS, Nin-like, AP2) and the T. ruralis homologues $\operatorname{Tr} 155$ [33, 41] and $\operatorname{Tr} 288$ [34, 41], TrDr3 [38], ELIPa [35], ALDH21 [37], Hsp70 and Lea 


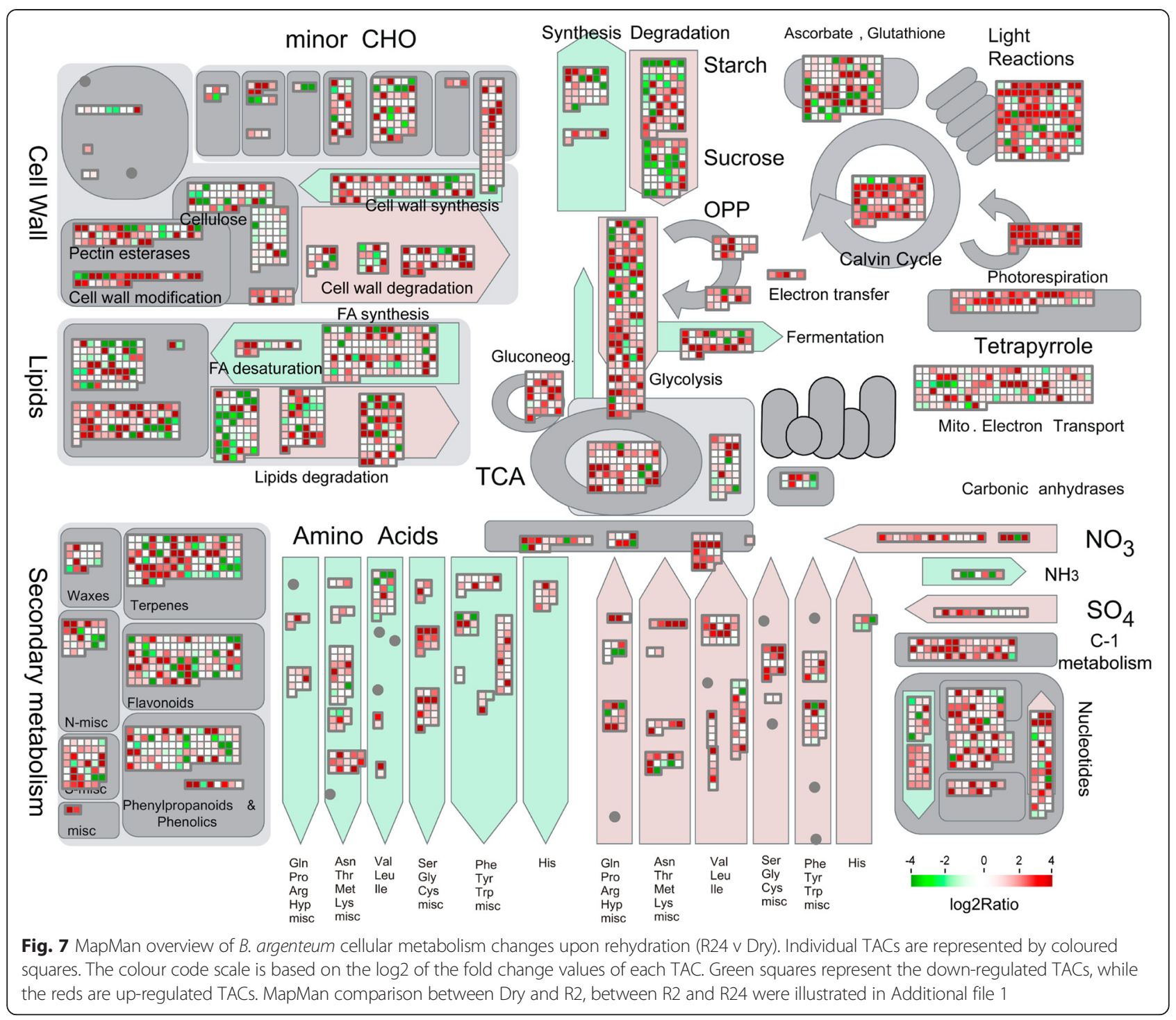

(Fig. 8). qPCR analysis of the transcripts confirmed the DGE expression data. In 6 of the transcripts (Hsp70, Aldh21, ERF, NAC, GRAS, Nin-like), including 4 of the 5 transcription factors, expression was elevated $2 \mathrm{~h}$ after hydration (relative to Dry) and declined $24 \mathrm{~h}$ after hydration. In 4 of the transcripts (AP2, Lea, Tr288 and TrDr3) expression was elevated at both Dry and R2 declined $24 \mathrm{~h}$ after rehydration. The rehydrin $\operatorname{Tr} 155$ was the only transcript with elevated expression $24 \mathrm{~h}$ after rehydration. In 5 of the 36 individual analyses, notable differences in the predicted versus validated expression amounts were observed: Dry Dr3 (Fig. 8g), Dry Tr155 (Fig. 8i), Dry LEA (Fig. 8j), Dry ELIPa (Fig. 8l) and R2 Tr288 (Fig. 8h). The rehydrin Tr155 and Tr288 were both first characterized in T. ruralis, Tr155 was postulated to be involved in antioxidant production during rehydration [33], and Tr288 possess a "K" segment common to dehydrins (a kind of LEA protein), which accumulated upon drying and declined over time following rehydration [34]. In our qPCR analysis of the two rehydrin homologs, Tr288 was detectable at R24 and Tr155 was slightly up-regulated after rehydration.

\section{Conclusions}

In this study we present a high-quality reference transcriptome for the desiccation-tolerant moss $B$. argenteum using next-generation, RNA-Seq technology. De novo transcriptome assembly generated 57,247 transcript assembly contigs (TACs). GO annotation of the $B$. argenteum transcriptome indicates the enhancement of sequence abundance related to 'envelope', 'biological regulation' and 'response to stimulus'. GO functional and pathway enrichment analysis of the DEGs demonstrated the induction of genes related to stress response and support the hypothesis that the rapid recovery of photosynthesis following rehydration, and de novo protein synthesis plays an important 

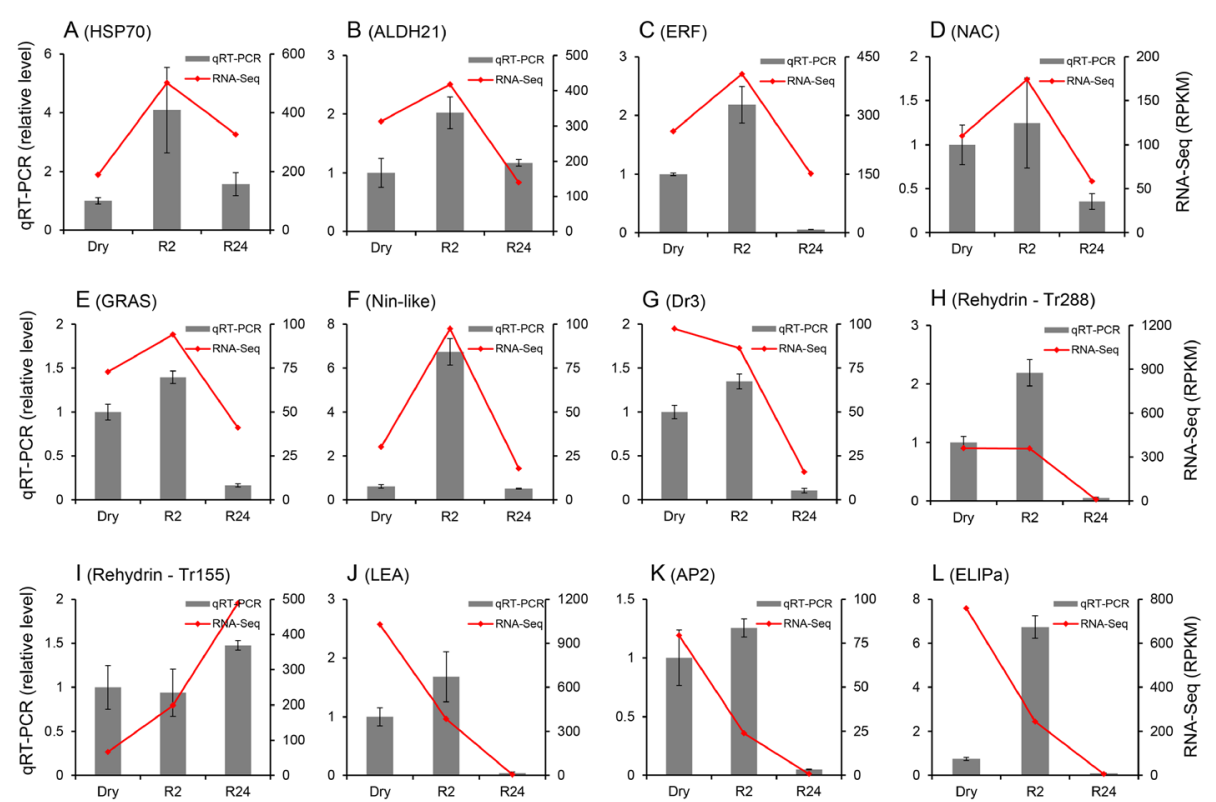

Fig. 8 qRT-PCR validation of selected B. argenteum TACs. Quantitative RT-PCR analysis of selected genes. Expression level of 12 genes were quantified using RT-qPCR, including HSP70 (a), aldehyde dehydrogenase 21 (b), ERF (c), NAC (d), GRAS (e) and Nin-like (f) transcription factors, homolog of T. ruralis desiccation-stress related gene (TrDr3) (g), 2T. ruralis rehydrin homologs (Tr288 (h) and Tr155 (i)), LEA (j), AP2 transcription factor (k) and Early Light Induced Protein (I). Quantitative gene expression data are shown as the mean \pm SD. The actin and tubulin genes were used as reference genes

role in the recovery of $B$. argenteum following rehydration. The data extends our knowledge of bryophyte transcriptomes and provides an insight into the molecular aspects of the rehydration process.

\section{Materials and methods}

\section{Plant material, culture and drying treatments}

Bryum argenteum Hedw. gametophores were collected from the Gurbantunggut Desert of Xinjiang Uygur Autonomous Region of China $\left(44^{\circ} 32^{\prime} 30^{\prime \prime} \mathrm{N}, 88^{\circ} 6^{\prime} 42^{\prime \prime} \mathrm{E}\right)$ and harvested and stored as described previously for Syntrichia caninervis gametophores [18]. Voucher specimens are maintained in the Department of Plant Biology, Southern Illinois University (Carbondale, IL, USA). $B$. argenteum gametophyte tissue was cultured on solid Knop media $(0.25 \mathrm{~g} / \mathrm{L} \mathrm{KH} 2 \mathrm{PO} 4, \mathrm{KCl}, \mathrm{MgSO} 4.7 \mathrm{H} 2 \mathrm{O}$, $1 \mathrm{~g} / \mathrm{L} \mathrm{Ca}(\mathrm{NO} 3) 2 \cdot 4 \mathrm{H} 2 \mathrm{O}$ and $0.0125 \mathrm{~g} / \mathrm{L} \mathrm{FeSO} 4.7 \mathrm{H} 2 \mathrm{O}$, $\mathrm{pH}$ adjusted to 5.8) in $9 \mathrm{~cm}$ Petri dishes. 60 explants were cultured at $25{ }^{\circ} \mathrm{C}$ with a $16 \mathrm{~h}$ photoperiod (under cool white fluorescent light, 4000 lux). Gametophytes of $B$. argenteum were prepared for experimentation as described [18]. Desiccated moss tissue was obtained by placing gametophytes on dry filter paper in an open Petri dish exposed to $20 \% \mathrm{RH}$ for $24 \mathrm{~h}$. This drying regime could result in the attainment of the gametophytes in desiccated state (Dry) that losses > $95 \%$ of its wet weight. Then the desiccated gametophytes (Dry) were rehydrated for $2 \mathrm{~h}$ (R2) with deionized water at $18{ }^{\circ} \mathrm{C}$ in the light. Samples were also taken for the fully rehydrated for $24 \mathrm{~h}$ (R24) gametophytes.

\section{RNA extraction, cDNA library construction and RNA sequencing}

For RNA sequencing, total RNAs were extracted using TRIzol reagent (Invitrogen, Carlsbad, CA, USA). The quality of total RNAs were checked using the NanoDrop 2000 Spectrometer and Agilent 2100 Bioanalyzer. High quality RNA samples were sent to Beijing Genomics Institute (BGI, Shenzhen, China) for cDNA libraries construction and sequencing using Illumina HiSeq 2000. For the purpose of this study, a two-step approach was applied to analyze the $B$. argenteum rehydration process. First, an integrated reference transcriptome was reconstructed and used for gene function annotation. The cDNA library for transcriptome generation was prepared by pooling the $B$. argenteum gametophores from different rehydration stages (Dry, R2 and R24). Second, we constructed 3 digital gene expression (DGE) libraries from different rehydration stages, including desiccated (Dry), rehydrated for $2 \mathrm{~h}$ (R2) and rehydrated for $24 \mathrm{~h}$ (R24) separately. The cDNA library construction method and Illumina deep-sequencing processes were the same as described previously [18, 45]. All the sequencing reads of the pooled transcriptome and the 3 DGE 
libraries have been deposited at the NCBI Sequence Read Archive (SRA) repository with accessions of SRR1763242, SRR1763243, SRR1763244 and SRR1763245.

\section{De novo transcriptome assembly and annotation}

Raw reads of low quality were eliminated or trimmed to generate clean reads, which were used for de novo transcriptome assembly using Trinity software [46], with optimized single k-mer length of 25. Then, the contigs generated by Trinity were further clustered with TGICL software [47] to get sequences (final transcript assembly contigs, TACs) that cannot be extended on either end, with parameters of "-1 40 -c 10 -v 20". Final reconstructed TACs with length equal to or longer than $200 \mathrm{bp}$ were retained for further analysis.

For annotating the $B$. argenteum transcriptome, TACs of the final assembly were searched against NCBI-nr, Swiss-prot, KEGG and COG databases using BLASTX with an E-value cut-off of 1e-5. Then all the TACs were divided into two groups: 'with BLAST' and 'without BLAST'. The coding region for each TAC and polypeptide sequences were then obtained using the method as described previously [18]. To evaluate the protein coding potential of the two groups of TACs, we used the program CPAT (Coding Potential Assessment Tool) v 1.2 [19], which calculates the coding potential for reconstructed transcripts. Furthermore, ORF size, GC content and expression levels were compared between the two sets of TACs. According to the NCBI-nr annotation results, the top BLASTX hits were used to identify putative homologous proteins and annotate each TAC with gene ontology (GO) terms using Blast2GO [48] with default parameters. Then the web-based program WEGO [49] was used to perform GO functional classification to illustrate the distribution of gene functional categories, according to molecular function, biological process, and cellular component ontologies.

To assess the completeness of the transcriptome assembly, all TACs were searched against the list of 357 Arabidopsis thaliana proteins that are conserved as single copy genes across all eukaryotes (i.e. ultra-conserved orthologs, UCOS, http://compgenomics.ucdavis.edu/compositae_reference.php), using BLASTX (E-value $\leq 1 \mathrm{e}-6$, and identity $\geq 30 \%$ ). To investigate metabolic pathway annotation, we performed a BLASTX of TACs against the KEGG database [22]; the resulting KEGG orthology and EC accession numbers were used to colour and retrieve the corresponding KEGG pathway maps.

For Pfam domain/family annotation, the predicted protein sequences were submitted to search against HMM profiles contained in the Pfam database (version 27.0) [23] using the software package HMMER v3.0 $[50,51]$. To resolve complex overlapping protein domains, only the most significant (lowest E-value) match within the clan was reported. The $B$. argenteum transcription factors were predicted using PlantTFDB v3.0 [24]. The putative transcription factors of $B$. argenteum were initially identified, including proteins that contain a DNA binding domain (inferred from Pfam annotation) or gave a positive BLASTP hit $($ E-value $\leq 1 \mathrm{e}-5)$ with recorded $P$. patens or $A$. thaliana transcription factors. Deduced polypeptide sequences were then submitted to the PlantTFDB prediction server (http://planttfdb.cbi.pku.edu.cn/prediction.php) for further classification and validation.

\section{Screening and analysis of DEGs}

All reconstructed TACs were used as the reference transcriptome sequences for the evaluation of the expression values. The $49 \mathrm{bp}$ reads sequenced from three individual DGE (Dry, R2 and R24) were mapped against the reference transcriptome with SOAP (v2.21), a short read aligner [52], with specific parameters of: " $-\mathrm{m} 0-\mathrm{x}$ 500 -s 40 -1 35 -v 5 -r 2". Mismatches of no more than two bases were allowed in the alignment. Expression level of B. argenteum TACs was normalized using RPKM method [25]. Rehydration regulated DEGs were identified with FDR $<0.001$ and absolute value of the $\log 2$ FoldChange $\geq 1[26,53]$.

The GO enrichment was analyzed with BiNGO plugin [39] in Cytoscape [54], using hypergeometric test for statistical analysis. For P-value correction, we selected the FDR correction method. GO terms with corrected $\mathrm{p}$-value $\leq 0.05$ were considered significantly over-representative and shown as colored nodes in the enrichment network. And significantly altered KEGG pathway were identified using a P-value based on hypergeometric distribution. All metabolic pathways with a FDR corrected P-value smaller than 0.05 were reported as significantly altered upon rehydration.

Furthermore, scrutiny of TAC expression changes was performed with MapMan $[55,56]$. The deduced polypeptide sequences were submitted to Mercator webserver [57] to classify them into MapMan functional plant categories. For color-coded representation (heat map) in MapMan, the $\log 2$ transformed of the fold-change for each TAC was employed. Deduced polypeptide sequences shorter than 50 amino acids were excluded to generate the MapMan metabolic pathway maps.

\section{Quantitative real-time PCR (qRT-PCR) analysis}

For quantitative real-time PCR, cDNA was synthesized using the PrimeScript ${ }^{\mathrm{m}} \mathrm{RT}$ reagent Kit (TaKaRa, China) according to the manufacturer's protocol with random hexamer primers. PCR primers were designed using the Primer Premier v5.0 software (Premier Biosoft, USA). The specificity of primer pairs were confirmed by BLASTN with non-redundant unigene set of $B$. argenteum transcripts, and melting curve analysis was performed for each primer 
pair before further analyses. qRT-PCR was performed using the QuantiFast SYBR Green PCR Kit (Qiagen, Germany) using a $20 \mu \mathrm{l}$ reaction volume following the manufacturer's protocol, fluorescence intensity was measured in the CFX96 $^{\text {ti }}$ Real-Time System (Bio-Rad, USA). Triplicates of each reaction were performed. The target gene expression levels were normalized using the actin and $\alpha$-tubulin genes as internal references. The relative abundance of transcript levels was calculated relative to the reference genes according to the $2^{-\Delta \Delta \mathrm{Ct}}$ method [58]. All data are shown as the mean \pm SD and all PCR primer information is provided in Additional file 1: Table S3.

\section{Additional files}

Additional file 1: Figure S1 - S10 and TableS1 - S3 mentioned in the article. Figure S1. Distribution of Ortholog Hit Ratios, calculated on the $B$. argenteum TACs with an positive BLASTX hit with P. patens. Figure S2. Venn diagram showing the BLASTX results of the $B$. argenteum TACs against four protein databases. Figure S3. Species distribution of the top BLASTX hits obtained using the $B$. argenteum transcriptome. Figure S4. Functional classifications of $\mathrm{GO}$ terms of $B$. argenteum TACs detected from Dry, R2 and R24. Figure S5. Differentially expressed B. argenteum TACs after $24 \mathrm{~h}$ rehydration of the photosynthesis metabolic pathway by KEGG annotation. Figure S6. Differentially expressed B. argenteum TACs after $24 \mathrm{~h}$ of rehydration of the photosynthesis - antenna proteins by KEGG annotation. Figure S7. Differentially expressed B. argenteum TACs after $24 \mathrm{~h}$ of rehydration of the ribosome proteins by KEGG annotation. Figure S8. MapMan overview of $B$. argenteum cellular metabolism changes following rehydrating $2 \mathrm{~h}$ (R2 $\vee$ Dry). Figure S9. MapMan overview of $B$. argenteum cellular metabolism changes between two rehydrated samples (R24 v R2). Figure S10. Hierarchical cluster analyses of the putative early light induced proteins following rehydration. Table S1. COG functional classification of B. argenteum TACs. Table S2. Statistically overrepresentative GO-slim terms of rehydration up-regulated genes in R2 and R24. Table S3. Primers used for quantitative real-time PCR (qRT-PCR) analysis.

Additional file 2: Complete list of $B$. argenteum TACs with BLASTX hits. Including BLASTX search hits in NCBI-Nr, Swiss-Prot, KEGG and COG databases.

Additional file 3: Pfam domain/family annotation of $B$. argenteum TACs and statistics of $B$. argenteum TACs per family (2 sheets).

Additional file 4: All B. argenteum transcription factors annotated using PlantTFDB and the lists of differentially expressed TFs (2 sheets).

Additional file 5: Digital gene expression profiles for Dry, R2 and R24 and the full list of differentially expressed genes by pairwise comparisons (4 sheets).

Additional file 6: Homologs of $T$. ruralis transcripts detected in $B$. argenteum and their expression RPKM values during rehydration.

Additional file 7: Significantly over-representative GO terms for up- and down-regulated genes. Hypergeometric test was performed for the DEGs to identify the over-representative GO terms using BiNGO. All the $\mathrm{GO}$ terms with a FDR corrected P-value less than 0.05 were shown in the tables ( 2 sheets).

\section{Abbreviations}

BSC: Biological soil crust; DEG: Differentially expressed genes; DGE: Digital gene expression; GO: Gene ontology; RPKM: Reads per kilobase per million mapped reads; TAC: Transcript assembly contig; TF: Transcription factor.

\section{Competing interests}

The authors declare that they have no competing interests.

\section{Authors' contributions}

BG performed the bioinformatics analyses, carried out the qPCR analysis and wrote the manuscript. DZ conceived the study and provided financial support for the project. XL contributed to the data analysis. HY participated in plant material preparations. YZ contributed to the discussion. AW contributed to data interpretation, revised and improved the manuscript. All authors read and approved the final manuscript.

\section{Acknowledgements}

This research was financially supported by the National Basic Research Program of China (2014CB954203) and the National Natural Science Foundation of China (U1170304)

\section{Author details}

${ }^{1}$ Key Laboratory of Biogeography and Bioresource in Arid Land, Xinjiang Institute of Ecology and Geography, Chinese Academy of Sciences, Urumqi 830011, China. ${ }^{2}$ University of Chinese Academy of Sciences, Beijing 100049, China. ${ }^{3}$ Department of Plant Biology, Southern Illinois University-Carbondale, Carbondale, IL 62901-6509, USA.

Received: 21 January 2015 Accepted: 18 May 2015

Published online: 28 May 2015

\section{References}

1. Bewley JD. Physiological Aspects of Desiccation Tolerance. Annu Rev Plant Physiol. 1979;30(1):195-238.

2. Wood AJ, Jenks MA: Plant desiccation tolerance: diversity, distribution, and real-world application. In: Plant Desiccation Tolerance. Blackwell Publishing Ltd; 2007:3-10.

3. Gaff DF, Oliver M. The evolution of desiccation tolerance in angiosperm plants: a rare yet common phenomenon. Funct Plant Biol. 2013;40(4):315-28.

4. Oliver M, Tuba Z, Mishler B. The evolution of vegetative desiccation tolerance in land plants. Plant Ecol. 2000;151(1):85-100.

5. Wood AJ. The nature and distribution of vegetative desiccation-tolerance in hornworts, liverworts and mosses. Bryologist. 2007;110(2):163-77.

6. Belnap J. The world at your feet: desert biological soil crusts. Front Ecol Environ. 2003;1 (4):181-9.

7. Zhang YM, Chen J, Wang L, Wang XQ, Gu ZH. The spatial distribution patterns of biological soil crusts in the Gurbantunggut Desert, Northern Xinjiang, China. J Arid Environ. 2007;68(4):599-610.

8. Li XR, Xiao HL, Zhang JG, Wang XP. Long-Term Ecosystem Effects of SandBinding Vegetation in the Tengger Desert. Northern China Restoration Ecology. 2004;12(3):376-90.

9. Li XR, He M-Z, Zerbe S, Li X-J, Liu L-C. Micro-geomorphology determines community structure of biological soil crusts at small scales. Earth Surf Process Landf. 2010;35(8):932-40.

10. Zheng Y, Xu M, Zhao J, Zhang B, Bei S, Hao L. Morphological Adaptations to Drought and Reproductive Strategy of the Moss Syntrichia caninervis in the Gurbantunggut Desert, China. Arid Land Research and Management. 2011;25(2):116-27.

11. Li J, Li X, Chen C. Degradation and reorganization of thylakoid protein complexes of Bryum argenteum in response to dehydration and rehydration. Bryologist. 2014;117(2):110-8.

12. Proctor MCF, Smirnoff N. Rapid recovery of photosystems on rewetting desiccation-tolerant mosses: chlorophyll fluorescence and inhibitor experiments. J Exp Bot. 2000;51(351):1695-704

13. Nabe H, Funabiki R, Kashino Y, Koike H, Satoh K. Responses to Desiccation Stress in Bryophytes and an Important Role of Dithiothreitol-Insensitive Non-Photochemical Quenching Against Photoinhibition in Dehydrated States. Plant Cell Physiol. 2007:48(11):1548-57.

14. Rensing SA, Lang D, Zimmer AD, Terry A, Salamov A, Shapiro H, et al. The Physcomitrella genome reveals evolutionary insights into the conquest of land by plants. Science. 2008;319(5859):64-9.

15. Zimmer A, Lang D, Buchta K, Rombauts S, Nishiyama T, Hasebe M, et al. Reannotation and extended community resources for the genome of the non-seed plant Physcomitrella patens provide insights into the evolution of plant gene structures and functions. BMC Genomics. 2013;14(1):498.

16. O'Neil S, Dzurisin J, Carmichael R, Lobo N, Emrich S, Hellmann J. Populationlevel transcriptome sequencing of nonmodel organisms Enynnis propertius and Papilio zelicaon. BMC Genomics. 2010;11(1):310. 
17. Szovenyi P, Perroud PF, Symeonidi A, Stevenson S, Quatrano RS, Rensing SA, et al. De novo assembly and comparative analysis of the Ceratodon purpureus transcriptome. Mol Ecol Resour. 2015;15(1):203-15.

18. Gao B, Zhang D, Li X, Yang H, Wood A. De novo assembly and characterization of the transcriptome in the desiccation-tolerant moss Syntrichia caninervis. BMC Research Notes. 2014;7(1):490.

19. Wang L, Park HJ, Dasari S, Wang S, Kocher JP, Li W. CPAT: Coding-Potential Assessment Tool using an alignment-free logistic regression model. Nucleic Acids Research 2013;41(6):e74.

20. Ferreira P, Patalano S, Chauhan R, Ffrench-Constant R, Gabaldon T, Guigo R, et al. Transcriptome analyses of primitively eusocial wasps reveal novel insights into the evolution of sociality and the origin of alternative phenotypes. Genome Biol. 2013;14(2):R20.

21. Derrien T, Johnson R, Bussotti G, Tanzer A, Djebali S, Tilgner $H$, et al. The GENCODE v7 catalog of human long noncoding RNAs: analysis of their gene structure, evolution, and expression. Genome Res. 2012;22(9):1775-89.

22. Kanehisa M, Goto S, Sato Y, Furumichi M, Tanabe M. KEGG for integration and interpretation of large-scale molecular data sets. Nucleic Acids Res. 2012;40(Database issue):D109-114.

23. Finn RD, Bateman A, Clements J, Coggill P, Eberhardt RY, Eddy SR, et al. Pfam: the protein families database. Nucleic Acids Res. 2014;42(Database issue):D222-230.

24. Jin J, Zhang H, Kong L, Gao G, Luo J. PlantTFDB 3.0: a portal for the functional and evolutionary study of plant transcription factors. Nucleic Acids Res. 2014;42(D1):D1182-7.

25. Mortazavi A, Williams BA, McCue K, Schaeffer L, Wold B. Mapping and quantifying mammalian transcriptomes by RNA-Seq. Nat Meth. 2008;5(7):621-8.

26. Audic S, Claverie J-M. The Significance of Digital Gene Expression Profiles. Genome Res. 1997;7(10):986-95.

27. Konishi M, Yanagisawa S. Arabidopsis NIN-like transcription factors have a central role in nitrate signalling. Nat Commun. 2013:4:1617.

28. Nakano T, Suzuki K, Fujimura T, Shinshi H. Genome-wide analysis of the ERF gene family in Arabidopsis and rice. Plant Physiol. 2006;140(2):411-32.

29. Hirsch S, Kim J, Munoz A, Heckmann AB, Downie JA, Oldroyd GE. GRAS proteins form a DNA binding complex to induce gene expression during nodulation signaling in Medicago truncatula. Plant Cell. 2009;21(2):545-57.

30. Hirsch S, Oldroyd GE. GRAS-domain transcription factors that regulate plant development. Plant Sigsnal Behav. 2009;4(8):698-700.

31. Oliver M, Dowd S, Zaragoza J, Mauget S, Payton P. The rehydration transcriptome of the desiccation-tolerant bryophyte Tortula ruralis: transcript classification and analysis. BMC Genomics. 2004;5(1):89.

32. Wood AJ, Duff RJ, Oliver MJ. Expressed sequence tags (ESTs) from desiccated Tortula ruralis identify a large number of novel plant genes. Plant Cell Physiol. 1999;40(4):361-8.

33. Oliver MJ, Wood AJ, O'Mahony P. How some plants recover from vegetative desiccation: A repair based strategy. Acta Physiol Plant. 1997;19(4):419-25.

34. Velten J, Oliver MJ. Tr288, a rehydrin with a dehydrin twist. Plant Mol Biol. 2001;45(6):713-22.

35. Zeng Q, Chen X, Wood AJ. Two early light-inducible protein (ELIP) CDNAs from the resurrection plant Tortula ruralis are differentially expressed in response to desiccation, rehydration, salinity, and high light. J Exp Bot. 2002;53(371):1197-205.

36. Chen X, Zeng Q, Wood AJ. Aldh7B6 Encodes a Turgor-Responsive Aldehyde Dehydrogenase Homologue That is Constitutively Expressed in Tortula ruralis Gametophytes. Bryologist. 2002;105(2):177-84.

37. Chen X. Zeng Qin, Wood AJ: The stress-responsive Tortula ruralis gene ALDH21A1 describes a novel eukaryotic aldehyde dehydrogenase protein family. J Plant Physiol. 2002;159(7):677-84.

38. Peng CA, Oliver MJ, Wood AJ. Is the Rehydrin TrDr3 from Tortula ruralis associated with tolerance to cold, salinity, and reduced $\mathrm{pH}$ ? Physiological evaluation of the TrDr3-orthologue, HdeD from Escherichia coli in response to abiotic stress. Plant Biol (Stuttg). 2005;7(3):315-20.

39. Maere S, Heymans K, Kuiper M. BiNGO: a Cytoscape plugin to assess overrepresentation of Gene Ontology categories in Biological Networks. Bioinformatics. 2005;21(16):3448-9.

40. Consortium GO. The Gene Ontology (GO) database and informatics resource. Nucleic Acids Res. 2004;32 suppl 1:D258-61.

41. Scott HB, Oliver MJ. Accumulation and polysomal recruitment of transcripts in response to desiccation and rehydration of the moss Tortula ruralis. J Exp Bot. 1994;45(5):577-83.
42. Wood AJ, Oliver MJ. Translational control in plant stress: the formation of messenger ribonucleoprotein particles (mRNPs) in response to desiccation of Tortula ruralis gametophytes. Plant J. 1999;18(4):359-70.

43. Li XP, Bjorkman O, Shih C, Grossman AR, Rosenquist M, Jansson S, et al. A pigment-binding protein essential for regulation of photosynthetic light harvesting. Nature. 2000;403(6768):391-5.

44. Alboresi A, Caffarri S, Nogue F, Bassi R, Morosinotto T. In Silico and Biochemical Analysis of Physcomitrella patens Photosynthetic Antenna: Identification of Subunits which Evolved upon Land Adaptation. PLoS One. 2008;3(4):e2033.

45. Zhao Z, Tan L, Dang C, Zhang H, Wu Q, An L. Deep-sequencing transcriptome analysis of chilling tolerance mechanisms of a subnival alpine plant, Chorispora bungeana. BMC Plant Biol. 2012;12(1):222.

46. Grabherr MG, Haas BJ, Yassour M, Levin JZ, Thompson DA, Amit I, et al. Full-length transcriptome assembly from RNA-Seq data without a reference genome. Nat Biotech. 2011;29(7):644-52.

47. Pertea G, Huang X, Liang F, Antonescu V, Sultana R, Karamycheva S, et al. TIGR Gene Indices clustering tools (TGICL): a software system for fast clustering of large EST datasets. Bioinformatics. 2003;19(5):651-2.

48. Conesa A, Götz S, García-Gómez JM, Terol J, Talón M, Robles M. Blast2GO: a universal tool for annotation, visualization and analysis in functional genomics research. Bioinformatics. 2005;21(18):3674-6.

49. Ye J, Fang L, Zheng H, Zhang Y, Chen J, Zhang Z, et al. WEGO: a web tool for plotting GO annotations. Nucleic Acids Res. 2006;34:W293-297. Web Server issue).

50. Eddy SR. A new generation of homology search tools based on probabilistic inference. Genome informatics International Conference on Genome Informatics. 2009;23(1):205-11.

51. Eddy SR. Accelerated Profile HMM Searches. PLoS Comput Biol. 2011;7(10):e1002195.

52. Li R, Yu C, Li Y, Lam T-W, Yiu S-M, Kristiansen K, et al. SOAP2: an improved ultrafast tool for short read alignment. Bioinformatics. 2009;25(15):1966-7.

53. Benjamini $Y$, Yekutieli $D$. The control of the false discovery rate in multiple testing under dependency. Annals of statistics 2001;29(4):1165-1188.

54. Smoot ME, Ono K, Ruscheinski J, Wang PL, Ideker T. Cytoscape 2.8: new features for data integration and network visualization. Bioinformatics. 2011;27(3):431-2.

55. Thimm O, Bläsing $O$, Gibon $Y$, Nagel A, Meyer $S$, Krüger $P$, et al. MAPMAN: a user-driven tool to display genomics data sets onto diagrams of metabolic pathways and other biological processes. Plant J. 2004;37(6):914-39.

56. Usadel B, Nagel A, Thimm O, Redestig H, Blaesing OE, Palacios-Rojas N, et al. Extension of the visualization tool MapMan to allow statistical analysis of arrays, display of corresponding genes, and comparison with known responses. Plant Physiol. 2005;138(3):1195-204.

57. Lohse M, Nagel A, Herter T, May P, Schroda M, Zrenner R, et al. Mercator: a fast and simple web server for genome scale functional annotation of plant sequence data. Plant, Cell Environ. 2014;37(5):1250-8.

58. Livak KJ, Schmittgen TD. Analysis of relative gene expression data using real-time quantitative PCR and the 2(-Delta Delta C(T)) Method. Methods. 2001;25(4):402-8.

\section{Submit your next manuscript to BioMed Central and take full advantage of:}

- Convenient online submission

- Thorough peer review

- No space constraints or color figure charges

- Immediate publication on acceptance

- Inclusion in PubMed, CAS, Scopus and Google Scholar

- Research which is freely available for redistribution

Submit your manuscript at www.biomedcentral.com/submit

C Biomed Central 